\title{
Fuzzy Square Difference Labeling of Some Graphs
}

Bharathi T*[1] Jeya Rowena ${ }^{2}$ and Ashwini Sibiya Rani $\mathrm{P}^{3}$

Received: 12 January 2021/ Accepted: 05 June 2021/ Published online: 18 June 2021

(C)Sacred Heart Research Publications 2017

\begin{abstract}
We introduced a new concept called the Fuzzy square difference labeling. We proved that the path graph $\left(P_{n}\right)$, the cycle graph $\left(C_{n}\right)$, the star graph $\left(S_{n}\right)$ and the complete bipartite graph $\left(K_{m, n}, n \leq 3\right)$ are Fuzzy square difference graphs.
\end{abstract}

Key words: Fuzzy Square Difference Labeling, Fuzzy Square Difference Graph.

\section{Introduction}

Fuzzy graph theory has numerous applications in many domains including networking, data mining, image capturing, communication, clustering, planning and scheduling. In comparison to fuzzy and classical models, fuzzy labeling models have more flexibility, precision and compatability to system. They also have many applications in Chemistry, Physics, Computer Science and other branches of Mathematics. The term Fuzzy logic was introduced by Lotfi Zadeh [7] in 1965. Fuzzy logic had also been studied by Lukasiewicz and Tarski [4]. It was Rosenfeld [5] who considered fuzzy relations on fuzzy sets and developed the theory of fuzzy graphs in 1975. He also studied the concept of fuzzy trees, blocks, bridges and cut nodes in fuzzy graphs. The concept of fuzzy labeling graphs was introduced by A. Nagoor Gani [2] 3], who studied the novel properties of fuzzy labeling graphs. The concept of Square difference labeling was introduced by J. Shiama [6]. He proved that many standard graphs like path, cycle, complete graph, wheels, comb, star, crown and some other graphs are square difference graphs.

\footnotetext{
${ }^{1,2,3} \mathrm{PG}$ and Research Department of Mathematics, Loyola College, University of Madras, Chennai, Tamil Nadu, India.
} Email: *prith.bharu23@gmail.com 
ISSN: 2456-8686, 5(1), 2021:070-075

https://doi.org/10.26524/cm93

\section{Preliminaries}

Definition 2.1 [5] A graph is a pair $(V, E)$ where $\mathrm{V}$ is a set and $\mathrm{R}$ is a relation on $\mathrm{V}$. Any fuzzy relation $\rho$ on a fuzzy subset $\mu$ of a set $\mathrm{V}$ can be regarded as a fuzzy graph where the edge $(u, v) \in V \times V$ has weight or strength $\rho(u, v) \in[0,1]$.

Definition 2.2 [6] A graph $G$ is said to have a Square difference labeling if there exist a bijection $f: V(G) \rightarrow\{0,1, \ldots, p-1\}$ such that the induced function $f^{*}: E(G) \rightarrow N$ given by $f^{*}(u v)=\left|(f(u))^{2}-(f(V))^{2}\right|$ for every $u v \in E(G)$ are all distinct. A graph which admits Square difference labeling is called a Square difference graph.

Definition 2.3 [1] A walk in a graph is a finite non-null subspace $W=v_{0} e_{1} v_{1} e_{2} v_{2}$ $\ldots e_{k} v_{k}$ whose terms are alternatively vertices and edges. we say that $W$ is a $\left(v_{0}, v_{k}\right)$ walk.

If the vertices of a walk $W$ are distinct, $W$ is called a path.

A closed trail whose origin and terminus are same is called a cycle.

Definition 2.4 [1] A star $S_{n}$ is the complete bipartite graph $K_{1, n}$, is a tree with one internal node and $n$ leaves.

Definition 2.5 [1] A graph $G$ is called bigraph or bipartite graph if $v$ can be partioned into two disjoint subsets $X$ and $Y$ such that every line of $G$ joins a point of $X$ to a point of $Y$. $(X, Y)$ is called a bipartition of $G$.

If further $G$ contains everyline joining the points of $X$ to the points of $Y$ then $G$ is called a complete bigraph or complete bipartite graph.

\section{Fuzzy square difference labeling of graphs}

Definition 3.1 A graph $G=(V, E)$ is said to be a Fuzzy square difference labeling if $\sigma: V \rightarrow[0,1]$ and $\mu: V \times V \rightarrow[0,1]$ are bijective and $\mu(u, v)<\sigma(u) \wedge \sigma(v)$ and $\mu(u, v)=\left|[\sigma(u)]^{2}-[\sigma(v)]^{2}\right|$ for every $(u, v) \in E$ are all distinct. A graph that admits Fuzzy square difference labeling is called Fuzzy square difference graph.

Theorem 3.2 Every Path graph $P_{n}$ is a Fuzzy square difference graph.

Proof: Let $\left\{v_{1}, v_{2}, v_{3}, \ldots, v_{n}\right\}$ be the set of vertices and $\left\{v_{1} v_{2}, v_{2} v_{3}, \ldots, v_{n-1} v_{n}\right\}$ be the set of edges. Choose $\omega \rightarrow(0,1]$ such that $\omega=10^{-1}$ for $n \leq 9$, $\omega=10^{-2}$ for 
ISSN: 2456-8686, 5(1), 2021:070-075

https://doi.org/10.26524/cm93

$10 \leq n \leq 99, \omega=10^{-3}$ for $100 \leq n \leq 999$ and so on.

$$
\begin{aligned}
\sigma\left(v_{i}\right) & =i * \omega, & & i=1,2, \ldots, n \\
\mu\left(v_{i}, v_{i+1}\right) & =3 * \omega^{2}, & & i=1 \\
\mu\left(v_{i}, v_{i+1}\right) & =2 * \omega^{2}+\mu\left(v_{i-1}, v_{i}\right), & & i=2,3, \ldots, n-1
\end{aligned}
$$

This labeling obey the conditions of Fuzzy square difference labeling. Thus every path graph is a Fuzzy square difference graph.

\section{Example 1.}

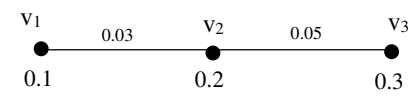

Figure 1: Fuzzy square difference labeling of $P_{3}$

Theorem 3.3 Every Cycle graph $C_{n}$ is a Fuzzy square difference graph.

Proof. Let $\left\{v_{1}, v_{2}, v_{3}, \ldots, v_{n}\right\}$ be the set of vertices and $\left\{v_{1} v_{2}, v_{2} v_{3}, \ldots, v_{n-1} v_{n}, v_{n} v_{1}\right\}$ be the set of edges. Choose $\omega \rightarrow(0,1]$ such that $\omega=10^{-2}$ for $n \leq 99, \omega=10^{-3}$ for $100 \leq n \leq 999, \omega=10^{-4}$ for $1000 \leq n \leq 9999$ and so on.

$\sigma\left(v_{i}\right)=i * \omega, \quad i=1,2, \ldots, n$

$\mu\left(v_{i}, v_{i+1}\right)=3 * \omega^{2}, \quad i=1$

$\mu\left(v_{i}, v_{i+1}\right)=2 * \omega^{2}+\mu\left(v_{i-1}, v_{i}\right), \quad i=2,3, \ldots, n-1$

$\mu\left(v_{i}, v_{1}\right)=\left(i^{2}-1\right) * \omega^{2}, \quad i=n$

This labeling obey the conditions of Fuzzy square difference labeling. Thus every cycle graph is a Fuzzy square difference graph.

\section{Example 2.}

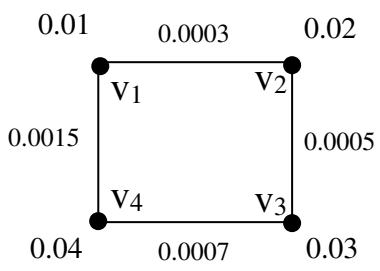

Figure 2: Fuzzy square difference labeling of $C_{4}$ 
ISSN: 2456-8686, 5(1), 2021:070-075

https://doi.org/10.26524/cm93

Theorem 3.4 Every Star graph $S_{n}$ is a Fuzzy square difference graph.

Proof. Let $\left\{v_{1}, v_{2}, v_{3}, \ldots, v_{n+1}\right\}$ be the set of vertices and $\left\{v_{1} v_{2}, v_{1} v_{3}, \ldots, v_{1} v_{n+1}\right\}$ be the set of edges.

Choose $\omega \rightarrow(0,1]$ such that $\omega=10^{-2}$ for $n \leq 99, \omega=10^{-3}$ for $100 \leq n \leq 999$, $\omega=10^{-4}$ for $1000 \leq n \leq 9999$ and so on.

$$
\begin{array}{rlrl}
\sigma\left(v_{i}\right) & =i * \omega, & i=1,2, \ldots, n+1 \\
\mu\left(v_{1}, v_{i+1}\right) & =3 * \omega^{2}, & i=1 \\
\mu\left(v_{1}, v_{i+1}\right) & =(2 i+1) * \omega^{2}+\mu\left(v_{1}, v_{i}\right), i=2,3, \ldots, n+1
\end{array}
$$

This labeling obey the conditions of Fuzzy square difference labeling. Thus every star graph is a Fuzzy square difference graph.

\section{Example 3.}

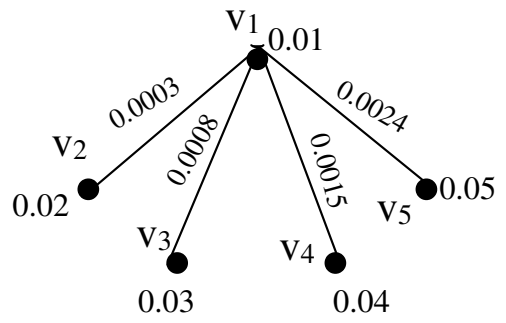

Figure 3: Fuzzy square difference labeling of $S_{4}$

Theorem 3.5 For $n \leq 3$, the Complete Bipartite graph $K_{n, m}$ is a Fuzzy square difference graph.

Proof. Let $\left\{v_{1}, v_{2}, v_{3}, \ldots, v_{n}, v_{n+1}, v_{n+2}, \ldots, v_{n+m}\right\}$ be the set of vertices and $\left\{v_{1} v_{n+1}, v_{1} v_{n+2}, \ldots, v_{1} v_{n+m}, v_{2} v_{n+1}, v_{2} v_{n+2}, \ldots, v_{2} v_{n+m}, v_{n} v_{n+1}, v_{n} v_{n+2}, \ldots, v_{n} v_{n+m}\right\}$ be the set of edges.

Choose $\omega \rightarrow(0,1]$ such that $\omega=10^{-2}$ for $n \leq 99, \omega=10^{-3}$ for $100 \leq n \leq 999$, $\omega=10^{-4}$ for $1000 \leq n \leq 9999$ and so on.

$\sigma\left(v_{i}\right)=i * \omega, i=1,2, \ldots, n, n+1, \ldots, n+m$

case $(\boldsymbol{i})$ : If $\mathrm{n}=1 \mu\left(v_{i}, v_{i+1}\right)=3 * \omega^{2}, \quad i=1$

$\mu\left(v_{1}, v_{i}\right)=(2 i-1) * \omega^{2}+\mu\left(v_{1}, v_{i-1}\right), \quad i=3,4, \ldots, n+m$ 
ISSN: 2456-8686, 5(1), 2021:070-075

https://doi.org/10.26524/cm93

$\operatorname{case}(\boldsymbol{i i}):$ If $\mathrm{n}=2$

$$
\begin{array}{rlrl}
\mu\left(v_{i}, v_{i+2}\right) & =8 * \omega^{2}, & & i=1 \\
\mu\left(v_{i+1}, v_{i+2}\right) & =5 * \omega^{2}, & & i=1 \\
\mu\left(v_{j}, v_{i}\right) & =(2 i-1) * \omega^{2}+\mu\left(v_{j}, v_{i-1}\right), & & j=1,2 \text { and } \\
& & & \\
& & i=4,5, \ldots, n+m
\end{array}
$$

case(iii): If $\mathrm{n}=3$

$$
\begin{array}{rlrl}
\mu\left(v_{i}, v_{i+3}\right) & =15 * \omega^{2}, & & i=1 \\
\mu\left(v_{i+1}, v_{i+3}\right) & =12 * \omega^{2}, & & i=1 \\
\mu\left(v_{i+2}, v_{i+3}\right) & =7 * \omega^{2}, & & i=1 \\
\mu\left(v_{j}, v_{i}\right) & =(2 i-1) * \omega^{2}+\mu\left(v_{j}, v_{i-1}\right), & & j=1,2,3 \text { and } \\
& & & \\
& & i=5,6, \ldots, n+m
\end{array}
$$

This labeling obey the conditions of Fuzzy square difference labeling. Thus the complete bipartite graph $K_{n, m}$ for $n \leq 3$ is a Fuzzy square difference graph.

\section{Example 4.}

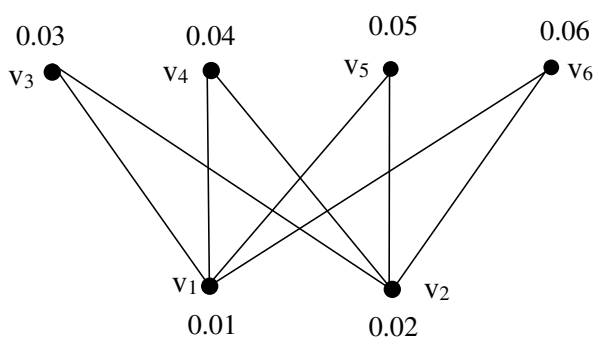

Figure 4: Fuzzy square difference labeling of $K_{2,4}$ 
ISSN: $2456-8686,5(1), 2021: 070-075$

https://doi.org/10.26524/cm93

\section{Conclusion}

We have studied the definition of Fuzzy square difference labeling and proved that path graph, cycle graph, star graph and complete graph $(n \leq 3)$ are Fuzzy square difference graphs.

\section{References}

[1] Arumugam S, Invitation to Graph Theory, Scitech Publications India Pvt Ltd., 2006.

[2] Gani AN, Akram M, Subahashini M, Novel Properties of fuzzy labeling graphs, Journal of Mathematics, 1-6 (2014) https://doi.org/10.1155/2014/375135.

[3] Gani AN, Subahashini, Properties of fuzzy labeling graph, Applied Mathematical Sciences, 6(70), 3461-3466 (2012).

[4] Pelletler, Francis Jeffry, Review of Metamathematics of fuzzy logics, The Bulletin of Symbolic Logic, 6(3), 342-346 (2000).

[5] Rosenfeld A, Fuzzy graphs, Fuzzy sets and their appllications, Academic Press, Newyork, 77-95, (1975).

[6] Shiama J, Some Special types of Square difference graphs, International Journal of Mathematical archives, 3(6), 2369-2374 (2012).

[7] Zadeh LA, Fuzzy sets, Information and Control, 8(3), 338-353 (1965). 